\title{
CISTECTOMIA RADICAL LAPAROSCÓPICA COM RESERVATÓRIO ILEAL
}

\author{
LAPAROSCOPIC RADICAL CYSTECTOMY WITH ILEAL RESERVOIR
}

\author{
Anibal Wood Branco'; William Kondo, ACBC-PR²; Alcides José Branco Filho²; \\ Luciano Carneiro Stunitz ${ }^{1}$; Marco Aurélio de George ${ }^{2}$
}

\section{INTRODUÇÃO}

A cistectomia radical é o tratamento padrão para os tumores malignos de bexiga, uroteliais ou mesenquimais, com invasão da camada muscular e sem evidência de metástase ${ }^{1,2}$. Com a maior aceitação da cirurgia laparoscópica para o tratamento das doenças do trato urinário superior, recentemente tem havido um maior interesse na cirurgia laparoscópica pélvica. Nos últimos anos, a cistectomia radical laparoscópica tem sido relatada com sucesso e segurança em centros de excelência em Urologia ${ }^{2-5}$, com as vantagens de apresentar menor perda sangüínea, redução da dor pós-operatória, retorno precoce às atividades habituais e ao trabalho, e melhor resultado estético quando comparada à cirurgia aberta ${ }^{1,2,5}$. No entanto, fatores como maior tempo cirúrgico, aumento dos custos e ausência de dados referentes ao resultado oncológico a longo prazo representam algumas das desvantagens dessa via de acesso minimamente invasiva ${ }^{3}$. O objetivo deste artigo é relatar nossa experiência inicial com a cistectomia radical com reservatório ileal por laparoscopia.

\section{RELATO DO CASO}

Paciente do sexo feminino, 29 anos, IMC de $23 \mathrm{~kg} / \mathrm{m}^{2}$, procurou nosso Serviço com queixa de hematúria macroscópica indolor. Foi submetida à cistoscopia que demonstrou a presença de lesão extensa em parede vesical à direita, cuja biópsia revelou um sarcoma de bexiga e a imunohistoquímica, um leiomiossarcoma.

Rx de tórax e tomografia computadorizada de abdome não evidenciaram lesão metastática. Não havia sinais de linfonodomegalia pélvica ou hidronefrose. A operação programada com o consentimento da paciente foi uma cistectomia radical, histerectomia e salpingoooforectomia esquerda laparoscópica com reservatório ileal. Teoricamente o ovário direito também deveria ser ressecado, mas foi preservado em decorrência da idade da paciente.

A paciente foi posicionada em decúbito dorsal com as pernas abduzidas, permitindo o acesso abdominal e perineal simultâneo. A mesa cirúrgica foi deixada em posição de
Trendelenburg a $45^{\circ}$. O acesso transperitoneal com cinco portais foi utilizado e o primeiro portal de $10 \mathrm{~mm}$ foi posicionado na linha média, $4 \mathrm{~cm}$ acima do umbigo, para a ótica de $0^{\circ}$. Os outros portais foram posicionados sob visão direta: $12 \mathrm{~mm}$ à direita e $10 \mathrm{~mm}$ à esquerda do umbigo, lateral ao músculo reto abdominal, e dois portais de $5 \mathrm{~mm}$ no quadrante inferior direito e esquerdo, aproximadamente $1 \mathrm{~cm}$ medial à espinha ilíaca ântero-superior ipsilateral.

Inicialmente foi realizada linfadenectomia pélvica extendida bilateral incluindo todo o tecido linfático entre o osso do púbis (distalmente) e a bifurcação da artéria ilíaca comum (proximalmente), e entre o nervo genitofemoral (lateralmente) e o nervo obturador (inferiormente). O peritônio do fundo de saco de Douglas foi incisado e a dissecção foi conduzida para a separação do reto. O pedículo vascular ovariano esquerdo, os ligamentos uterinos, as artérias uterinas e o pedículo vascular vesical foram ligados. O espaço de Retzius foi dissecado e a bexiga foi mobilizada anteriormente incluindo o úraco. A parede posterior da vagina foi incisada horizontalmente logo distalmente à cérvice uterina utilizando eletrocautério. Esta incisão foi estendida bilateralmente em direção à uretra, removendo uma porção da parede anterior da vagina. A uretra foi suturada para não ocorrer extravasamento de urina na cavidade abdominal.

O espécime cirúrgico foi retirado da cavidade em bloco através da vagina (Figura 1). O pneumoperitônio foi re-estabelecido e mantido através da colocação de uma compressa úmida no canal vaginal. A vagina foi fechada laparoscopicamente utilizando sutura contínua de vicryl $2-0$.

O íleo distal foi identificado e um segmento de $15 \mathrm{~cm}$ de íleo foi isolado utilizando grampeador linear cortante $45 \mathrm{~mm}$. O trânsito intestinal foi reconstruído através de anastomose látero-lateral também com grampeador linear cortante $45 \mathrm{~mm}$. $\mathrm{O}$ ureter esquerdo foi passado retroperitonealmente para o lado direito. Os ureteres foram implantados na alça de íleo através de anastomose término-lateral com pontos contínuos, com um cateter duplo J no interior. A extremidade distal da alça ileal foi levada para fora da cavidade abdominal através da ampliação do orifício do portal de $12 \mathrm{~mm}$ para-retal di-

1. Urologista do Hospital da Cruz Vermelha, Curitiba - PR.
2. Cirurgião Geral do Hospital da Cruz Vermelha, Curitiba - PR.
Recebido em 25/01/2006
Aceito para publicação em 20/03/2006
Conflito de interesses: nenhum
Fonte de financiamento: nenhuma
Trabalho realizado no Departamento de Urologia e de Cirurgia Geral do Hospital da Cruz Vermelha, Curitiba, PR. 


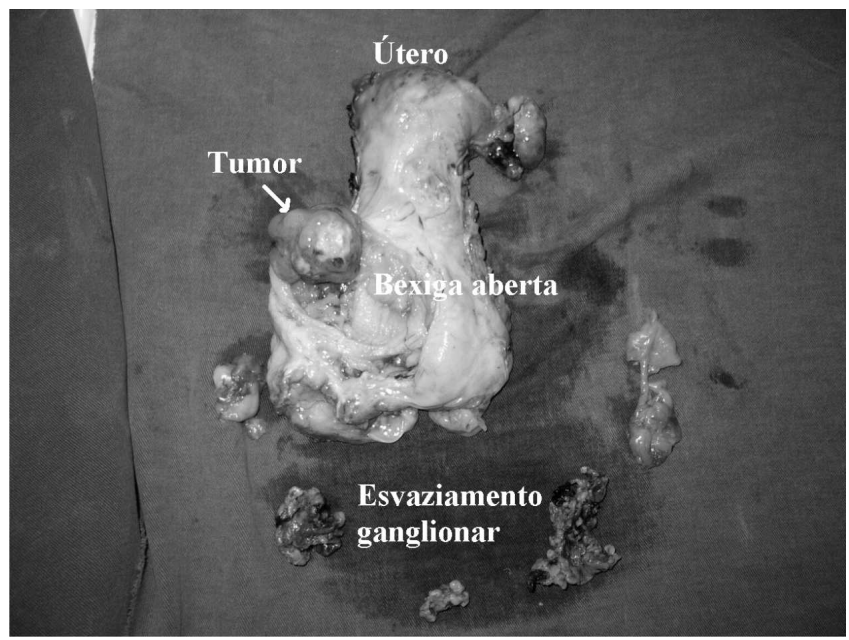

Figura 1 - Peça cirúrgica removida via vaginal.

reito. Um dreno túbulo laminar foi posicionado através do orifício do trocater de $5 \mathrm{~mm}$ da fossa ilíaca esquerda.

O tempo cirúrgico total foi de 405 minutos com sangramento intra-operatório de $500 \mathrm{ml}$. Não houve nenhuma intercorrência no intra-operatório. A evolução pós-operatória foi satisfatória. Dieta líquida foi iniciada no $2^{\circ}$ dia após a cirurgia, o dreno de penrose foi retirado no $4^{\circ}$ dia e a paciente recebeu alta hospitalar no $5^{\circ}$ dia. Os valores da hemoglobina e da creatinina sérica no momento da alta eram, respectivamente, $10,5 \mathrm{~g} / \mathrm{dl}$ e $0,8 \mathrm{mg} / \mathrm{dl}$. Seis meses após a cirurgia, a creatinina sérica é de $0,9 \mathrm{mg} / \mathrm{dl}$, a radiografia de tórax e a tomografia computadorizada de abdome e pelve não evidenciam metástase e a urografia excretora apresenta excreção renal bilateral normal.

A patologia revelou um leiomiossarcoma de bexiga de baixo grau com margens cirúrgicas negativas e ausência de comprometimento de linfonodos da cadeia ilíaca-obturadora.

\section{DISCUSSÃO}

A primeira cistectomia laparoscópica foi descrita em 1992 e desde então a experiência em todo o mundo tem crescido $^{4}$, com diversas variações técnicas tendo sido publicadas nos últimos anos ${ }^{1-5}$. No Brasil, os primeiros casos foram descritos em 2005, utilizando a técnica vídeoassistida $^{3}$.

Os benefícios da via de acesso laparoscópica já estão bem estabelecidos e está claro que a cistectomia radical laparoscópica é um procedimento factível ${ }^{5}$. No entanto, é uma operação bastante complexa e exige grande habilidade técnica; provavelmente este seja um fator limitante e talvez o principal fator responsável pela lenta disseminação do procedimento no meio urológico. Mesmo em mãos experientes, o tempo cirúrgico permanece longo, em grande parte devido ao tempo necessário para a reconstrução urinária ${ }^{1}$. Atualmente, um dos focos principais dos estudos é a busca de métodos para reduzir o tempo necessário para essa reconstrução ${ }^{5}$, seja a realizando intra ou extra-corpórea, por meio de laparoscopia pura ou vídeo-assistida.

O pequeno número de pacientes e a curta duração do acompanhamento ainda não permitem conclusões a respeito de resultados a longo prazo. Séries maiores com seguimento oncológico futuramente poderão elucidar dados referentes à sobrevida após a cirurgia por laparoscopia ${ }^{1}$.

Neste artigo pudemos demonstrar o primeiro caso de cistectomia radical com reservatório ileal totalmente laparoscópica em nossa instituição. Acreditamos que a cirurgia minimamente invasiva realmente tem um papel importante na era cirúrgica atual, principalmente reduzindo a morbidade aos pacientes, e deve ser encorajada a todos os grupos com grande experiência em laparoscopia.

\begin{abstract}
Worldwide experience with laparoscopic radical cystectomy is increasing in the last few years. We describe a 29-year-old female who underwent a laparoscopic radical cystectomy with ileal reservoir for a bladder sarcoma. Operative time was 405 minutes and estimated blood loss was $500 \mathrm{~mL}$. She had an uneventful postoperative course and was discharged in the fifth postoperative day. Key-words: bladder, cystectomy, laparoscopy, urinary diversion, bladder neoplasms (Rev. Col. Bras. Cir. 2008; 35(2): 149-150).
\end{abstract}

Key words: Urinary Bladder; Cystectomy; Laparoscopy/methods; Urinary Diversion; Urinary Bladder Neoplasms.

\section{REFERÊNCIAS}

1. Hrouda D, Adeyoju AA, Gill IS. Laparoscopic radical cystectomy and urinary diversion: fad or future? BJU Int. 2004; 94(4):501-5.

2. Taylor GD, Duchene DA, Koeneman KS. Hand assisted laparoscopic cystectomy with minilaparotomy ileal conduit: series report and comparison with open cystectomy. J Urol. 2004; 172(4 Pt 1):1291-6.

3. Abreu SC, Silveira RA, Cerqueira JB, Regadas RP, Gonzaga LF, Fonseca GN. Stapleless laparoscopic assisted radical cystectomy with ileal neobladder in a male and with ileal loop in a female: initial report from Brazil. Int Braz J Urol. 2005; 31(3):214-20.

4. Moinzadeh A, Gill IS, Desai M, Finelli A, Flacone T, Kaouk J. Laparoscopic radical cystectomy in the female. J Urol. 2005; 173(6):1912-7.
5. Moinzadeh A, Gill IS. Laparoscopic radical cystectomy with urinary diversion. Curr Opin Urol. 2004;14(2):83-7.

\section{Como citar este artigo:}

Branco AW, Kondo W, Branco Filho AJ, Stunitz LC, George MA. Cistectomia radical laparoscópica com reservatório ileal - relato de caso. Rev Col Bras Cir. [periódico na Internet] 2008; 35(2). Disponível em URL: http://www.scielo.br/rcbc

Endereço para correspondência:

Anibal Wood Branco

Rua das Palmeiras, 170 ap. 201

80620-110 - Curitiba - PR

E-mail: anibal@awbranco.com.br 